\title{
The Evolution of Tumors in Mice and Humans with Germline p53 Mutations
}

\author{
Arnold J. Levine, ${ }^{1,2}$ Chang S. Chan, ${ }^{2,3}$ Crissy Dudgeon, ${ }^{2}$ Anna \\ Puzio-Kuter, ${ }^{2}$ and Pierre Hainaut ${ }^{4}$ \\ ${ }^{1}$ Institute for Advanced Study, Princeton, New Jersey 08540 \\ ${ }^{2}$ Cancer Institute of New Jersey, New Brunswick, New Jersey 08903 \\ ${ }^{3}$ Department of Medicine, Rutgers Robert Wood Johnson Medical School, New Jersey 08903 \\ ${ }^{4}$ Grenoble Institute for Research on Cancer, Grenoble 38706, France \\ Correspondence: alevine@ias.edu
}

\begin{abstract}
Mice with a homozygous 53 gene deletion develop thymic lymphomas by 9 wk of age. Using the sequence of the rearranged T-cell receptor gene from each clone of cells in the thymus, one can determine the number of independent transformation events. These tumors are oligoclonal, occurring at a frequency of $0.13-0.8$ new cancer clones per day. By 20 wk only a few clones are detected, indicating competition among transformed cell clones. DNA sequencing of these tumors demonstrates a point mutation frequency of one per megabase and many genes that are consistently amplified or deleted in independent tumors. The tumors begin with an inherited p53 gene deletion. Next is a PTEN mutation in a stem cell or progenitor cell, before the rearrangement of the T-cell receptor. After that, the T-cell clone selects gene amplifications in cyclin D and cdk-6, and in Ikaros in the Notch pathway. Humans heterozygous for the p53 mutant gene in the germline ( $\mathrm{Li}-$ Fraumeni syndrome) develop cancers at an early age. The penetrance of heterozygous p 53 mutations is $\sim 93 \%$ of individuals developing tumors over their lives. At older ages the remaining $7 \%$ of this $\mathrm{Li}$-Fraumeni population actually have a lower risk of developing tumors than the population at large with wild-type p53 genes.
\end{abstract}

More than $50 \%$ of all human cancers contain mutations in both alleles of the p53 gene, and individuals with germline mutations in this gene develop $\mathrm{Li}-$ Fraumeni syndrome, a disorder with increased risk of developing cancers, especially at young ages (Hollstein et al. 1991; Malkin 2011). The p53 protein is a transcription factor that responds to a wide variety of stresses by initiating transcriptional programs for cell cycle arrest and DNA repair, apoptosis, and senescence. The stresses that activate p53-mediated transcription include DNA damage, hypoxia, nutritional deprivation, spindle damage, aneuploidy, interference with proper ribosomal biogenesis, telomere shortening, and mutational activation of selected oncogenes (Levine and Oren 2009). The replication of cells in the presence of any of these stresses leads to an enhanced mutation frequency. The transcriptional activation of p53 is thought to result in the elimination of clones of cells carrying these mutations. p53 knockout mice develop $\mathrm{CD}^{4+} / \mathrm{CD}^{8+} \mathrm{T}$-cell thymic lymphomas by 2-6 mo after birth (Donehower et al. 1995), but the events leading to these tumors remain largely unexplored. We do not know the mutation frequencies of these tumors during their development and over time. It is unclear whether these tumors are initiated as a single clone that evolves with time or whether the tumor is composed of several independent or oligoclonal lineages that could compete with each other for resources. How diverse is the mutational spectrum of the tumor? Do the same oncogenes and inactive tumor-suppressor genes drive $\mathrm{p} 53^{-}$- thymic lymphomas formed in independent mice? If that is the case, are the mutations that arise in these tumors selected for in a specific order of events after the p53 mutation is inherited? Do some of these mutations occur in stem cells, and are they selected for in the stem cell compartments of the T-cell precursors before T-cell differentiation? All of these questions are addressed and answered in this study.

Based upon these results, it will become clear that there is an order to the selection of some mutations in $\mathrm{p} 53^{-/-}$ stem or early T-cell-lineage progenitors. The absence of a p53 protein enhances the mutation frequency of these cells, producing oligoclonal tumors, which display high levels of copy number variations (CNVs; deletions, amplifications, and aneuploidy). Surprisingly, there are not an excessive number of point mutations. Additional mutations result in the evolutionary selection for a smaller number of clones that survive and are detectable with time. These results will be compared with epidemiological observations of human $\mathrm{Li}$-Fraumeni families with heterozygous p53 mutations. These studies suggest that developmental lineages (mesenchymal and ectodermal

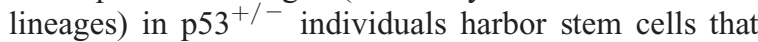
are much more at risk for developing tumors from certain tissues at specific young ages, when those tissues are actively replicating. In contrast, individuals who inherit wild-type p53 genes and who develop tumors derived from endodermal derivatives contain high numbers of somatic p53 mutations in both alleles. Finally, the penetrance for tumor formation in p53 mutant $\mathrm{Li}$-Fraumeni families is $\sim 93 \%$, with $\sim 7 \%$ of $\mathrm{p} 53^{+/-}$individuals es- 
caping clinically observable tumors during their lifetimes. Interestingly, these individuals actually have a lower risk of developing cancers after 70-75 yr of age than do $\mathrm{p} 53^{+/+}$individuals in the population. This could be the result of good luck, a good environment, or the presence of modifier alleles of the p53 genes in their genomes.

\section{RESULTS AND DISCUSSION}

\section{Thymic Lymphomas in $\mathbf{p 5 3} 3^{-/-}$Mice}

$\mathrm{p} 53^{-/-}$mice develop thymic lymphomas over the first 6 mo of life. As each T cell is produced in the thymus it acquires a unique $\mathrm{T}$-cell receptor. This permits one to determine the progeny of a clonal lineage of a $\mathrm{T}$ cell by sequencing the V-D-J region of the DNA from a rearranged T-cell receptor gene (Robins et al. 2009; Gopalakrishnan et al. 2013). The number of sequence reads of identical V-D-J DNA sequences (modified by terminal transferase) is proportional to the number of offspring from a T-cell clone. To establish a baseline for the normal T-cell levels in wild-type mice, the DNA from the thymus of wild-type C57Bl/ 6 mice was extracted from two males and from two females at embryonic day 17 (E17), 3, 6, 9, and $20 \mathrm{wk}$ after birth. The polymerase chain reaction (PCR) primers were localized just adjacent to the $\mathrm{V}$ region and $\mathrm{J}$ region $(\sim 200 \mathrm{nt}$ after T-cell receptor [TCR] rearrangements are produced) of the TCR- $\beta$ chain gene. A possible PCR primer bias that can alter the number of copies that are amplified and sequenced was corrected for by employing algorithms provided by Adaptive Biotechnologies (Carlson et al. 2013). The number of sequence reads permits one to calculate the number of unique clones and replicated clones of T-cell progeny in the thymus. Table 1 provides the percentage of $\mathrm{T}$ cells from the two most common clones and their progeny in the thymus. The highest frequencies of replicated clones from the wild-type C57Bl/6 mouse (males and females) varied between $0.08 \%$ and $0.17 \%$ of the total T cells se-

Table 1. Percentage of the two largest clones in WT and $\mathrm{p} 53-\mathrm{KO}$ mice

\begin{tabular}{lcr}
\hline Time & $\mathrm{wT}$ & $\mathrm{p} 53-\mathrm{KO}$ \\
\hline E17 & $0.16 \%, 0.15 \%$ & $0.16 \%, 0.14 \%$ \\
& $0.17 \%, 0.17 \%$ & $0.15 \%, 0.13 \%$ \\
& $0.17 \%, 0.14 \%$ & $0.25 \%, 0.20 \%$ \\
3 wk & $0.16 \%, 0.15 \%$ & $0.19 \%, 0.18 \%$ \\
& $0.10 \%, 0.05 \%$ & $0.07 \%, 0.07 \%$ \\
& $0.07 \%, 0.07 \%$ & $0.18 \%, 0.14 \%$ \\
6 wk & $0.07 \%, 0.05 \%$ & \\
& $0.06 \%, 0.05 \%$ & $0.10 \%, 0.10 \%$ \\
9 wk & $0.08 \%, 0.08 \%$ & $0.11 \%, 0.11 \%$ \\
& $0.16 \%, 0.13 \%$ & \\
20 wk & $0.11 \%, 0.11 \%$ & $2.9 \%, 1.2 \%$ \\
& $0.08 \%, 0.07 \%$ & \\
& $0.07 \%, 0.06 \%$ & $70 \%, 28 \%$ \\
& $0.16 \%, 0.14 \%$ & $43 \%, 19 \%$ \\
& $0.12 \%, 0.11 \%$ & $70 \%, 5 \%$ \\
& $0.15 \%, 0.09 \%$ & $43 \%, 19 \%$ \\
\hline
\end{tabular}

WT, wild type; p53-KO, p53 knockout. quenced (average of $0.11 \% \pm 0.05 \%$ ) at different times before and after birth. In the p53 $3^{-/}$thymus (Table 1 ), the maximum number of DNA reads from T-cell clones remained the same from E17 to $6 \mathrm{wk}$ after birth (Student $t$ test for a difference; $P=0.14 \pm 0.054)$. By 9 wk after birth, in the $\mathrm{p} 53^{-/-}$mice, the number of progeny from highest clones rose (30- to 200-fold) as shown in Table 1, and by $20 \mathrm{wk}$ the most common T-cell clones were 120 - to 600-fold higher in p53 mutant mice than in wild-type mice. These statistically significant increases in T-cell clones and their progeny were used as the definition that a thymic lymphoma was produced. This was confirmed histologically. The increased number of progeny from a clone is not due to antigen exposure in the thymus. The ratio of productive (in reading frame) to nonproductive TCR- $\beta$ chains produced was similar over the first 6 -wk period of life in both wild-type and $\mathrm{p} 53^{-/-}$mice, indicating that the loss of p53 did not interfere with this process. However, as the oligoclonal tumors began to be produced at 9 and $20 \mathrm{wk}$ the ratio of productive clones $\left(\mathrm{TCR}^{+}\right)$to nonproductive clones $\left(\mathrm{TCR}^{-}\right)$was reduced four- to fivefold.

The number of different clones of $\mathrm{T}$ cells contributing to a thymic lymphoma in a single $\mathrm{p} 53^{-/-}$mouse showed that multiple independent transformation events occurred and the tumors are oligoclonal. By $20 \mathrm{wk}$, dominant clones arose, so that in one case $98 \%$ of the clones were composed of two different clones ( $70 \%$ and $28 \%$ ). In another case as many as 10 different clones contributed to the tumor (Fig. 1). The number of T-cell lymphomas produced in a mouse between 9 and 20 wk averaged between 0.13 and 0.8 transformed clones per day. The fact that thymic lymphomas were not detected until 9 wk of age suggested that additional mutations must contribute to the formation of these lymphomas. The loss of the p53 gene was not sufficient to produce a thymic lymphoma.

\section{Whole-Exome Sequencing of $\mathrm{p53}^{-/-}$ Thymic Lymphomas}

Whole-exome sequencing of three thymic lymphomas at $20 \mathrm{wk}$ after birth was performed. A matched tail DNA from one wild-type C57Bl/6 mouse was used as a control sequence. The nonsynonymous point mutations and frameshift/stop codon mutations detected by this sequencing uncovered no reproducible tumor driver mutations in the three tumor samples sequenced. The mutation frequency was about one mutation per megabase, which is not exceptionally high when compared with human breast or pancreatic tumor frequencies (Lawrence et al. 2013). There was no indication that the absence of p53 in these mice contributes to excessive point mutations when compared with a large number of p53 wildtype or mutant tumors.

\section{Copy Number Variations (CNVs) in p53 Knockout Thymic Lymphomas}

The exome sequencing permitted the reconstruction of CNVs that were found in all three of the tumors 
A

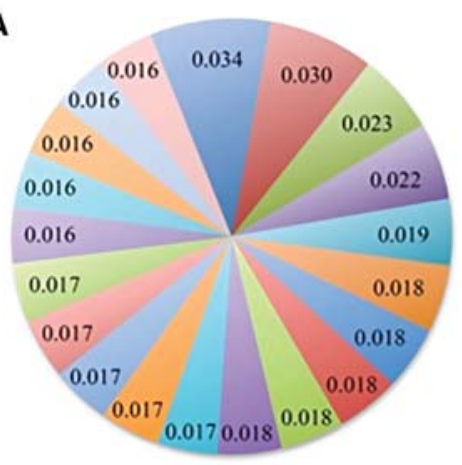

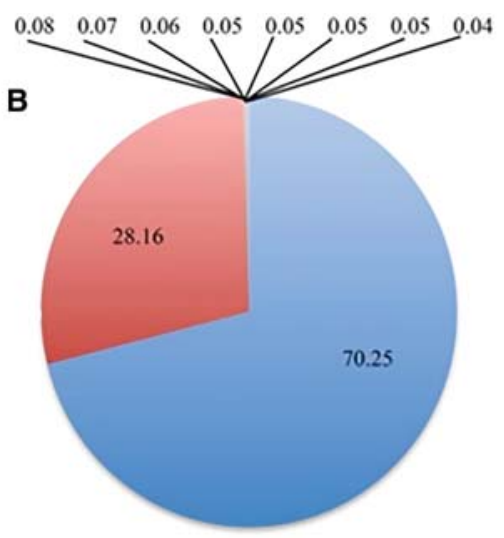

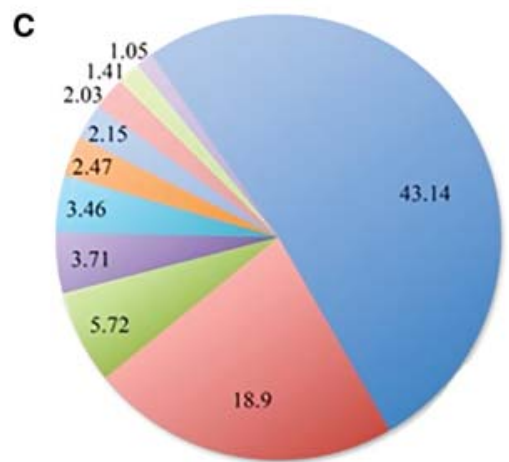

Figure 1. p53 knockout (p53-KO) thymic lymphomas are oligoclonal and comprised of a few dominant clones. $(A)$ Clones from a wild-type thymus: The frequencies of the top 10 clones for each normal T-cell detected. $(B, C)$ The most frequent clones detected in p53 knockout mice were plotted to show dominant clones where in one case $(B) 98 \%$ of the clones were composed of two different clones $(70 \%$ and $28 \%)$ and in another case $(C) 10$ different clones contributed to the tumor. Both tumors are from male p53-KO mice.

sequenced. For deletions in the genome, a cutoff of 0.3 was used, corresponding to $38 \%$ of the tumor sample having a one-copy deletion. For amplifications, a 0.2 increase was used, corresponding to $30 \%$ of the tumor sample having a one-copy amplification. Based upon these criteria, the three tumors had 276,348 , and $422 \mathrm{CNVs}$, respectively. In contrast with the point mutations, these $\mathrm{CNVs}$ produced a reproducible pattern of losses and gains in the three genomes compared. An example of one of these tumors is shown in Figure 2. Chromosome 3 of this mouse genome has a chromothripsis signature, which is commonly observed in human tumors with $\mathrm{p} 53$ mutations and appears to arise by extensive recombination, resulting in alternate gains and losses on a chromosome. It is of some interest that biallelic Pten deletions are detected in all three of the independent mouse tumors. Each of these three mice had different Pten deletions. However, within an individual mouse all of the Pten deletions detected by sequencing were identical, even though the tumors were oligoclonal, with diverse origins. For example, in one mouse, the tumor was composed of clones representing $39.4 \%, 13.5 \%, 8.4 \%$, and $7.0 \%$ of the total tumor load, but $85 \%$ of the sequence reads for the Pten deletion have identical exons deleted. In a second mouse the tumor was composed of two clones of $76.7 \%$ and $9.7 \%$, and $98 \%$ of the Pten DNA reads were identical. These data are most consistent with the hypothesis that the Pten deletion occurred before the TCR rearrangement in a stem or early T-cell lineage progenitor. The Pten deletion, along with the inherited p 53 loss, contributes to future tumors that arise from these clones. Different $\mathrm{p} 53^{-/-}$mice have different Pten deletions, but in each mouse the deletion arises and is selected for early in development so that all tumors in a given mouse have the same mutation. Many chromosomal aneuploidies were detected, and chromosome 5 was amplified in all three sequenced tumors. The patterns of selected genome rearrangements were surprisingly reproducible with $\sim 175$ genes recurrently amplified in all tumors sequenced. These amplifications occurred in a smaller percentage of clones that produce lymphomas, suggesting that they occurred after the TCR rearrangement. A comparison of the amplified or altered genomes from different mice commonly detected Cdk6 and Ikzf1 (Ikaros) as two genes whose amplifications are also observed in human T-cell lymphomas and may drive these tumors.

\section{Pten, Cyclin D, Cdk6, and Ikaros Expression in Thymic Lymphomas}

The results of the CNVs observed by DNA sequencing were confirmed by western blots of Pten, Cdk6, cyclin $\mathrm{D} 1-3, \mathrm{Rb}$, and Ikaros (Ik 1-3) and its dominant-negative splice variants (Ik4-8). Antibodies to each of these proteins were compared in western blots employing tissues from wild-type mice and $\mathrm{p} 53^{-/-}$mice that were taken at 6 wk of age (before detection of a tumor) and compared with samples of thymic lymphomas taken from 10 different mice at $20 \mathrm{wk}$ of age. The results are presented in Figure 3. The Pten proteins are lost from seven of the 10 tumors and a reduced level of Pten protein was observed in the three other tumors. The Cdk6 kinase is overexpressed in all 10 tumors and either cyclin D1, D2, and/ or D3 (or combinations of the D cyclins) are overexpressed in all of the tumors. The retinoblastoma protein $\mathrm{Rb}$ is deleted in three tumors. Dominant-negative forms of the Ikaros proteins (Ik4-8) are expressed in nine of the 10 tumors, and the Ik protein is missing in the 10th tumor. Ikaros is in the Notch pathway of T-cell development, and these types of mutations have been observed in human T-cell lymphomas (Chilosi et al. 1998; Sun et al. 1999; Gutierrez et al. 2009).

The inherited $\mathrm{p} 53^{-/-}$genotype results in genomic instability and rapidly selects in the stem or progenitor cells of T cells for Pten mutations, which contribute a metabolic preparation for rapid growth. Pten mutations alter glucose metabolism (the Warburg effect) in these cells. After the rearrangements of the T-cell receptor, the amplifications of the Cdk6 gene and one or more of the cyclin D genes help to promote rapid cell cycle replication. Then 
chr1

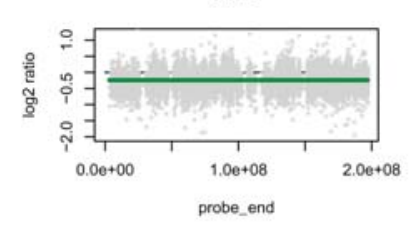

chr6

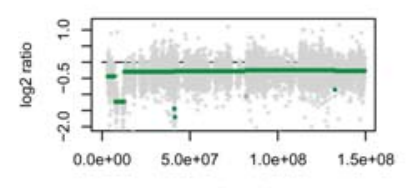

probe_end

chr11

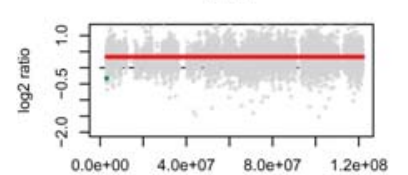

probe_end

chr16

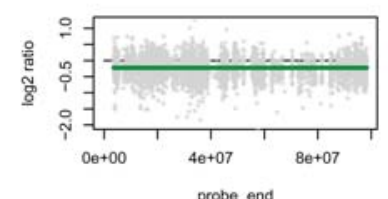

chr2

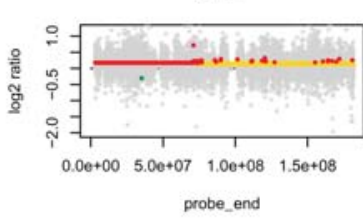

chr7

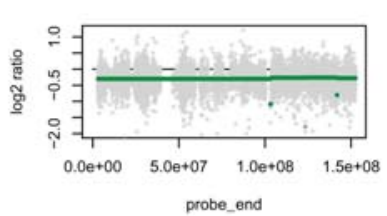

chr12

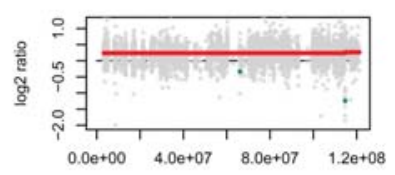

probe_end

chr17

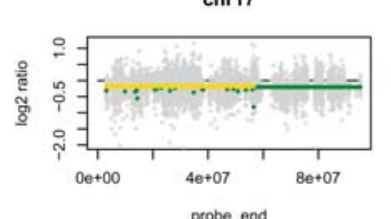

chr3

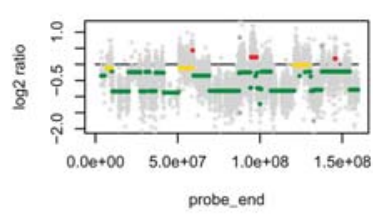

chr8

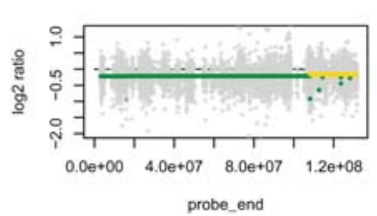

chr13

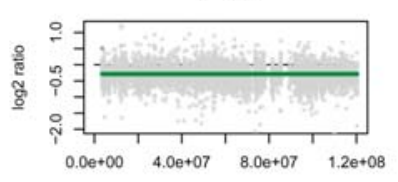

probe_end

chr18

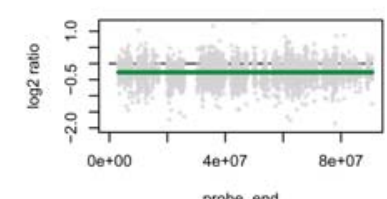

chr4

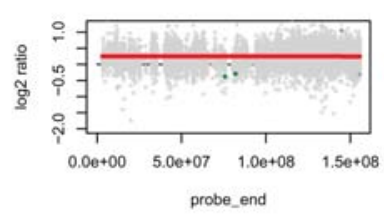

probe-ent

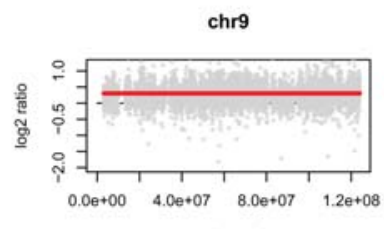

probe_end

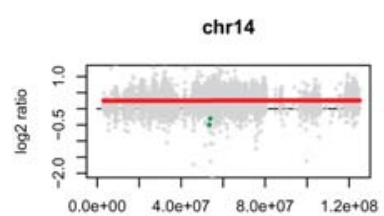

probe_end

chr19

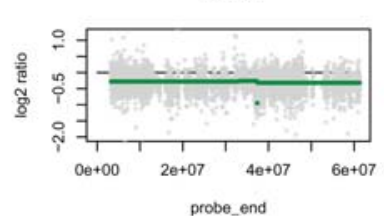

chr5

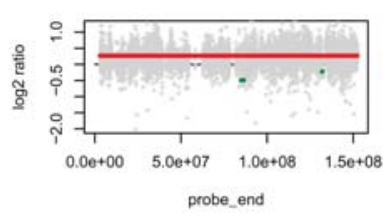

chr10

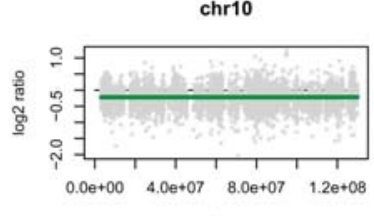

probe_end

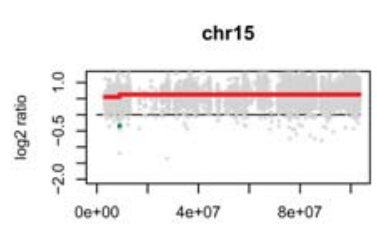

probe_end

Figure 2. p53 knockout ( p53-KO) thymic lymphomas have increased copy number variations (CNVs). CNV plots are from one male p53 knockout thymic lymphoma. Red lines show areas of amplification, yellow lines show no change, and green lines are areas of deletion. 


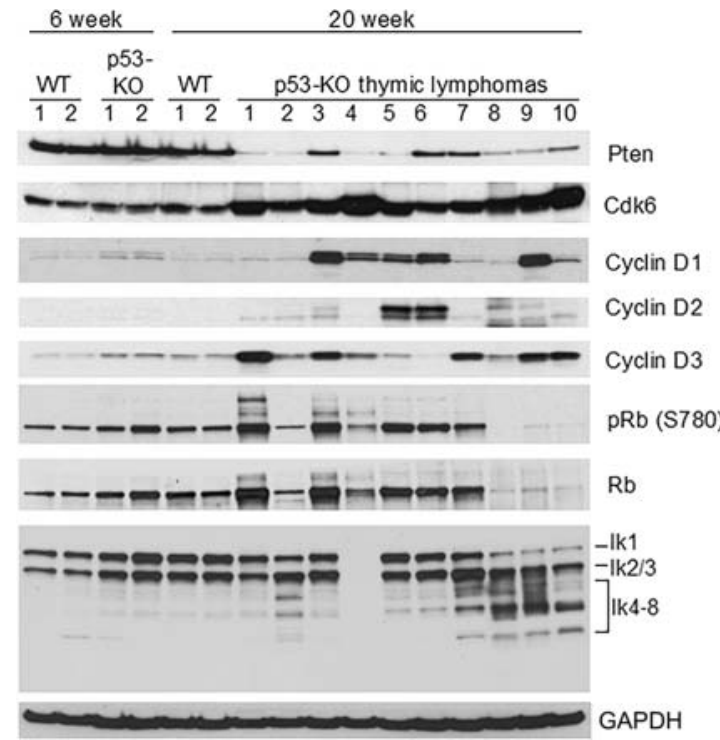

Figure 3. CNV results confirmed by western blots of p53 knockout ( $\mathrm{p} 53-\mathrm{KO})$ thymic lymphomas. Western blot analysis of 6wk-old wild-type (WT), 6-wk-old p53-KO thymus, and 20-wkold p53-KO thymic lymphoma proteins show Pten loss, cyclin $\mathrm{D}$, and Cdk6 overexpression and Ik4-8 dominant-negative expression. Proteins analyzed include Pten, Cdk6, cyclin D1, cyclin D2, cyclin D3, phospho-Rb, Rb, and Ikaros isoforms (Ik18). GAPDH (glyceraldehyde-3-phosphate dehydrogenase) was used as a loading control.

Ikaros mutations block development of differentiated T cells, producing CD4/8 double-positive T-cell lymphomas. These results suggest that once a p53 mutation is inherited, it may act as a gatekeeper mutation, resulting in the order of selection of subsequent mutations (such as Pten) producing larger numbers of clones that form the precursors for additional mutations at a higher frequency and in tumor formation at an early age. The epidemiological evidence from humans with $\mathrm{Li}$-Fraumeni syndrome and inherited p53 mutations supports this notion.

\section{Germline p53 Mutations in Humans}

A relatively small number of humans and families harbor p53 mutations in the heterozygous state, which in $\sim 70 \%$ of the cases results in $\mathrm{Li}-$ Fraumeni syndrome (Olivier et al. 2010). This is an autosomal-dominant disorder that results in a subset of tumors commonly expressed at a young age of onset. Patients may develop multiple independent cancers and have a lifetime risk of 93\% for developing a tumor. In most cases, a single mutant p53 allele is inherited (or arises spontaneously) and the wild-type allele is commonly (but not always) lost during tumor initiation or formation. The International Association of Cancer Research (IARC, Lyon, France) collects and stores a great deal of information about $\mathrm{Li}$-Fraumeni families. The analysis of these data was performed by Pierre Hainaut from the R16 version of the IARC database (November 2012). It describes 634 families with p53 germline mutations producing 2483 cancer cases with a 30,000 person-year follow-up. One of the first observations made with the database was that individuals who inherit p53 mutations develop cancers during their lifetime that derive from the mesenchymal and ectodermal lineages at a five- to 100 -fold greater risk than observed in the wild-type population. The risk of developing a tumor derived from the endodermal lineage is only two- to threefold higher than in the wild-type population. This is in contrast to the observation that somatic p53 mutations are found at a very high frequency in endodermal tumors in the colon $(75 \%)$, lung (75\%), pancreas $(80 \%)$, and ovary $(100 \%)$. There is clearly a difference in the impact of a p53 mutation acquired by inheritance rather than as a somatic mutation, despite the fact that the same mutant alleles are observed in both inherited and somatic p53 mutations. The developmental origin of the tissue affects the p53-mediated risk of tumor formation (Fig. 4). A second, similar observation, deduced from these data is that the tissue specificity and the type of tumor produced in a $553^{+/-}$carrier differ depending upon the chronological age of the patient. Adrenocortical carcinomas (ADCs) occur at a 50- to 100 -fold risk in patients between the ages of 6 mo to 4 yr compared with wild-type populations. Choroid plexus papillomas (CPTs) occur at 50- to 100-fold increased risk between 6 mo and $3 \mathrm{yr}$, whereas medullary blastomas (MEDs) occur at 50- and 100-fold increased risk between 2 and $9 \mathrm{yr}$. Rhabdomyosarcomas are observed at a 50- to 100-fold increased risk between 1 and $4 \mathrm{yr}$ after birth. Leiomyosarcomas are produced between 20 and $60 \mathrm{yr}$ of age, liposarcomas between 1 and $50 \mathrm{yr}$ of age, and breast tumors at $18-45$ yr of age in $\mathrm{Li}-$ Fraumeni patients, all at five- to 10-fold higher risk than in the wild-type population (Fig. 5). Rather clearly, the ages at which a tumor arises in individuals with a $53^{+/-}$genotype reflects the time of most active tissue cell division and the activity of tissue-specific stem cells in the mesenchymal and ectodermal lineages (but not the endodermal lineages). These data tie p53 function to selected germline tissue types and different stages in the development of the organism. These observations also distinguish between inherited (acting early in development) and somatic (acting later in tumor formation) p53 mutations. This study suggests a role for p53 in selected stem cells, rapid cell division, and tissue development over a lifetime.

The epidemiology of this syndrome also uncovers the presence of a small group of patients $(\sim 7 \%)$ who by $65-100 \mathrm{yr}$ of age have never developed a clinically active tumor, even though they have a $553^{+/-}$genotype, with an allele that will result in tumors in other families or individuals in a family. These individuals actually have a lower risk of developing a cancer than the wild-type population in the same age group. This may be explained by good luck, by living in a terrific environment that does not stress the patient, or by the presence of suppressor alleles that increase haploid levels of p53 activity and protect the patient. Enhanced protein modifications or levels of the wild-type p53 protein, inhibition of the negative regulator of p53 (MDM2), or alleles that back up or replace some p53 functions might all be considered as viable candidates for this type of genetic modifier. 


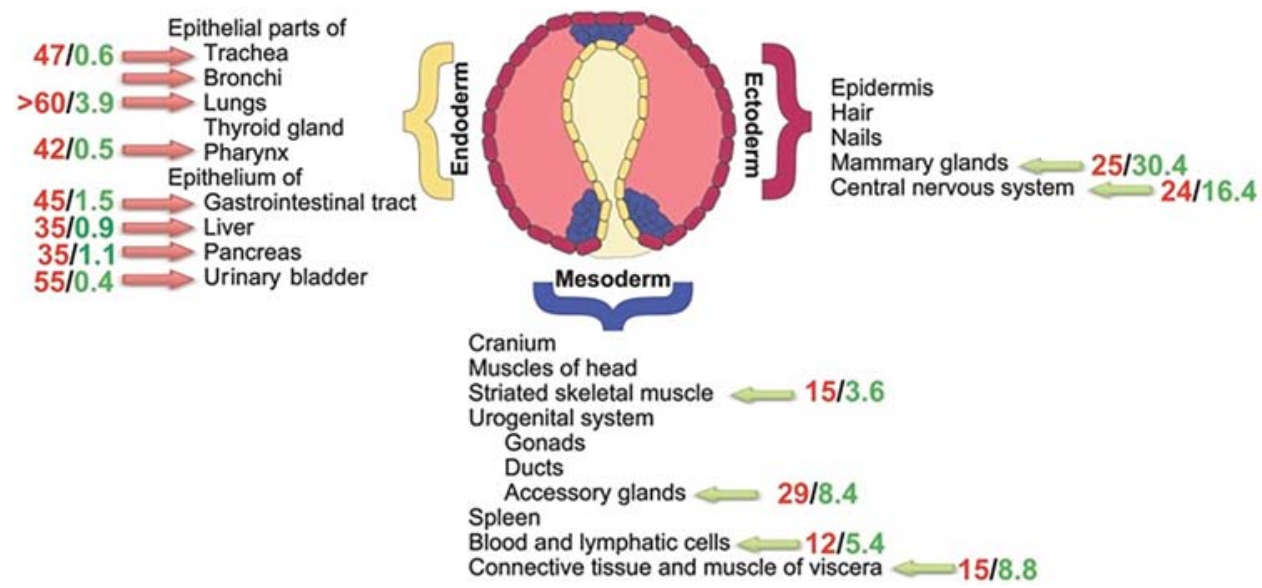

Figure 4. Distinct embryonic origin for tumors with high rates of somatic TP53 mutations and tumors frequently associated with germline TP53 mutations. Red arrow indicates tissues/organs in which tumors with high rates of TP53 mutations $(>35 \%)$ occur (\% somatic mutations: numbers in red). Green arrows indicate tissues/organs in which tumors frequently develop in subjects who carry a germline TP53 mutation (\% of all cancer diagnoses in mutation carriers: numbers in green). Tumors that are frequent in mutation carriers are different from those which often contain TP53 somatic mutations, with the exception of cancers of the lung and colorectum.

\section{MATERIALS AND METHODS}

Mice

All animal protocols used in this study were approved by the Rutgers Biomedical and Health Sciences Animal Care and Use Committee. WT and p53-KO mice were killed at the indicated times using $\mathrm{CO}_{2}$ and thymus tissue placed in liquid nitrogen for future analysis.

\section{DNA Extraction and TCR- $\beta$ Sequencing}

Thymus DNA was extracted using the PureLink Genomic DNA Kit (Life Technologies, Carlsbad, CA). The TCR- $\beta$ locus was sequenced using the ImmunoSEQ survey level assay by Adaptive Biotechnologies (Seattle,
WA) from WT and p53-KO mice at various ages as well as p53-KO thymic lymphomas. TCR- $\beta$ sequencing was analyzed using the ImmunoSEQ Analyzer (Adaptive Biotechnologies).

\section{Exome Sequencing and Somatic Point Mutation Analysis}

Whole-exome sequencing and SNP/Indel calling was completed by BGI Americas, Inc. (Philadelphia, PA). The exonic regions were captured using Agilent SureSelect Mouse All Exon $50 \mathrm{Mb}$ and sequenced by 100bp paired-end reads on an Illumina platform. Somatic point mutation analysis was completed as previously shown (Dudgeon et al. 2014). Somatic point mutations

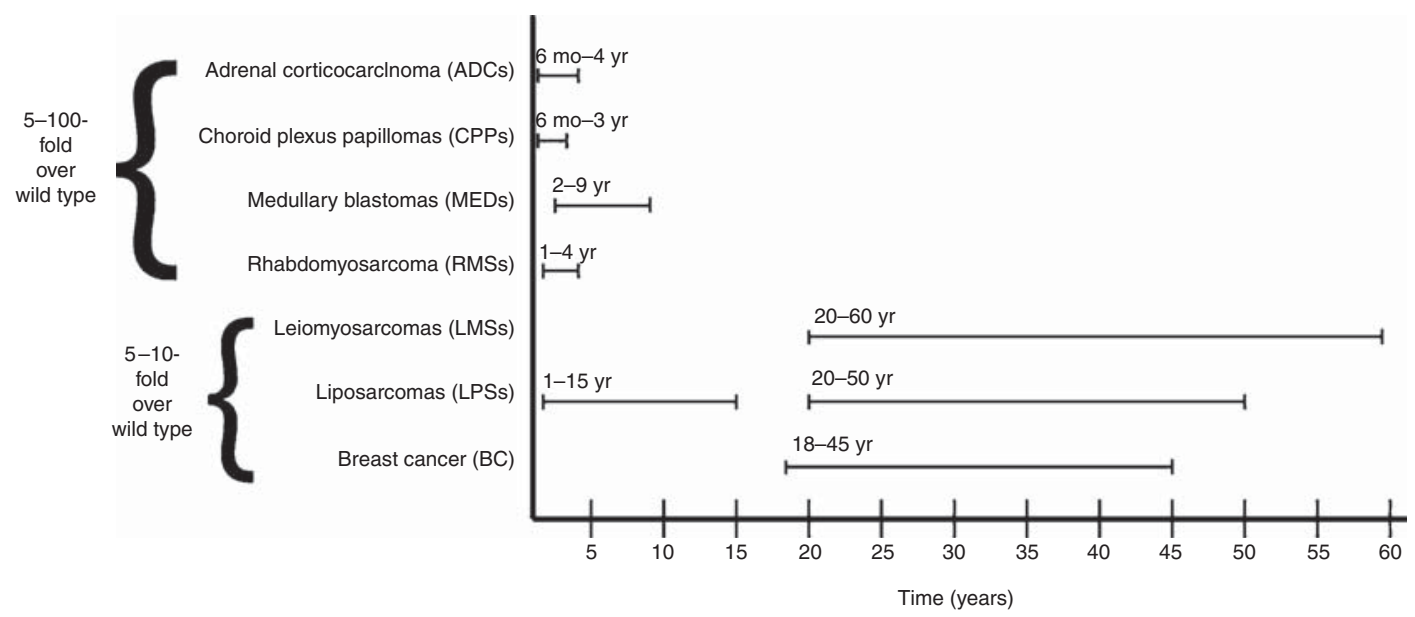

Figure 5. Differences in tumor type and tissue specificity in p53 heterozygous carriers is dependent on chronological age. ADCs occur between the ages of 6 mo to $4 \mathrm{yr}$, whereas CPTs occur between 6 mo and 3 yr of age, MEDs between 2 and 9 yr of age, RMSs between 1 and $4 \mathrm{yr}$, LMSs between 20 and $60 \mathrm{yr}$ of age, LPSs between 1 and $50 \mathrm{yr}$ of age, and breast tumors at $18-45 \mathrm{yr}$ of age in Li-Fraumeni patients. The indicated tumor types occur most often in tissues that replicate and develop in a time-dependent fashion over a lifetime. 
were called on matched normal and tumor BAM files using MuTect (Cibulskis et al. 2013). After identifying somatic genomic variants, known single-nucleotide polymorphisms (SNPs) were identified by comparing with dbSNP128 for mouse. After filtering known SNPs, somatic mutations were found in tumor samples as compared with normal sample.

\section{Structural Variation Analysis}

CNV analysis was accomplished using ExomeCNV (Sathirapongsasuti et al. 2011) using the default parameter setting with sample admixture rate set to 0.5 . Analysis was completed as described (Dudgeon et al. 2014).

\section{Western Blotting}

Twenty micrograms of protein extract from thymus and thymic lymphomas was subjected to SDS-PAGE (sodium dodecyl sulfate-polyacrylamide gel electrophoresis) and transferred to an Immobilon-P membrane (EMD Millipore, Billerica, MA). Western blot was performed using antibodies to Pten (\#9188), Cdk6 (\#3136), cyclins D1 (\#2978), D2 (\#3741), D3 (\#2936), phospho-Rb (S780) (\#9307), Rb (\#9313), Ikaros (\#5443) (Cell Signaling, Danvers, MA), and Gapdh (\#25778) (Santa Cruz Biotechnology, Inc., Dallas, TX).

\section{REFERENCES}

Carlson CS, Emerson RO, Sherwood AM, Desmarais C, Chung MW, Parsons JM, Steen MS, LaMadrid-Herrmannsfeldt MA, Williamson DW, Livingston RJ, et al. 2013. Using synthetic templates to design an unbiased multiplex PCR assay. Nat Commun 4: 2680.

Chilosi M, Doglioni C, Yan Z, Lestani M, Menestrina F, Sorio C, Benedetti A, Vinante F, Pizzolo G, Inghirami G. 1998. Differential expression of cyclin-dependent kinase 6 in cortical thymocytes and T-cell lymphoblastic lymphoma/leukemia. Am J Pathol 152: 209-217.
Cibulskis K, Lawrence MS, Carter SL, Sivachenko A, Jaffe D, Sougnez C, Gabriel S, Meyerson M, Lander ES, Getz G. 2013. Sensitive detection of somatic point mutations in impure and heterogeneous cancer samples. Nat Biotechnol 31: 213-219.

Donehower LA, Harvey M, Vogel H, McArthur MJ, Montgomery CA Jr, Park SH, Thompson T, Ford RJ, Bradley A. 1995 Effects of genetic background on tumorigenesis in p53-deficient mice. Mol Carcinog 14: 16-22.

Dudgeon C, Chan C, Kang W, Sun Y, Emerson R, Robins H, Levine AJ. 2014. The evolution of thymic lymphomas in p53 knockout mice. Genes Dev 28: 2613-2620.

Gopalakrishnan S, Majumder K, Predeus A, Huang Y, Koues OI, Verma-Gaur J, Loguercio S, Su AI, Feeney AJ, Artyomov MN, et al. 2013. Unifying model for molecular determinants of the preselection V $\beta$ repertoire. Proc Natl Acad Sci 110: E3206-E3215.

Gutierrez A, Sanda T, Grebliunaite R, Carracedo A, Salmena L, Ahn Y, Dahlberg S, Neuberg D, Moreau LA, Winter SS, et al. 2009. High frequency of PTEN, PI3K, and $A K T$ abnormalities in T-cell acute lymphoblastic leukemia. Blood 114: 647-650.

Hollstein M, Sidransky D, Vogelstein B, Harris CC. 1991. p53 mutations in human cancers. Science 253: 49-53.

Lawrence MS, Stojanov P, Polak P, Kryukov GV, Cibulskis K, Sivachenko A, Carter SL, Stewart C, Mermel CH, Roberts SA, et al. 2013. Mutational heterogeneity in cancer and the search for new cancer-associated genes. Nature 499: 214-218.

Levine AJ, Oren M. 2009. The first 30 years of p53: Growing ever more complex. Nat Rev Cancer 9: 749-758.

Malkin D. 2011. Li-Fraumeni syndrome. Genes Cancer 2: 475-484.

Olivier M, Hollstein M, Hainaut P. 2010. TP53 mutations in human cancers: Origins, consequences, and clinical use. Cold Spring Harb Perspect Biol 2: a001008.

Robins HS, Campregher PV, Srivastava SK, Wacher A, Turtle CJ, Kahsai O, Riddell SR, Warren EH, Carlson CS. 2009. Comprehensive assessment of T-cell receptor $\beta$ chain diversity in $\alpha \beta$ T cells. Blood 114: 4099-4107.

Sathirapongsasuti JF, Lee H, Horst BA, Brunner G, Cochran AJ, Binder S, Quackenbush J, Nelson SF. 2011. Exome sequencing-based copy-number variation and loss of heterozygosity detection: ExomeCNV. Bioinformatics 27: 2648-2654.

Sun L, Crotty ML, Sensel M, Sather H, Navara C, Nachman J, Steinherz PG, Gaynon PS, Seibel N, Mao C, et al. 1999. Expression of dominant-negative Ikaros isoforms in T-cell acute lymphoblastic leukemia. Clin Cancer Res 5: 21122120 . 


\section{$\overbrace{\text { CSH\& }}^{\infty}$ Cold Spring Harbor Symposia SYMPOSIA On Quantitative Biology}

\section{The Evolution of Tumors in Mice and Humans with Germline p53 Mutations}

Arnold J. Levine, Chang S. Chan, Crissy Dudgeon, et al.

Cold Spring Harb Symp Quant Biol 2015 80: $139-145$ originally published online December 7, 2015

Access the most recent version at doi:10.1101/sqb.2015.80.027631

References This article cites 15 articles, 7 of which can be accessed free at: http://symposium.cshlp.org/content/80/139.full.html\#ref-list-1

\section{License}

Email Alerting Service the box at the top right corner of the article or click here. 